\title{
Effet d'amorçage structurel en français langue seconde : une étude de corpus longitudinale
}

\author{
Thomas, Anita \\ Centre de Langues et Littérature \\ Université de Lund \\ Suède \\ anita.thomas@rom.lu.se
}

\section{Introduction}

L'influence de l'input sur l'acquisition d'une langue seconde (L2) a été largement reconnue durant les quinze dernières années (Ellis 2002 ; Véronique 2010), mais les recherches sur la nature de cette influence restent limitées. Les études effectuées dans le cadre interactionniste (Gass 1997, 2003 ; Long 1983, 1996 ;) soulignent le rôle bénéfique de l'interaction sur le développement d'une L2, parce qu'un tel contexte mobilise un certain nombre de processus psychologiques tels que l'attention ou le noticing tout en permettant des activités tels que des feedbacks, des recasts ou des négotiations de sens. Comme le suggère Gass (1997), l'input devient saillant dans le dialogue (voir aussi Bozier 2005 ; Matthey 1996 et les séquences potentiellement acquisitionnelles). Les études effectuées dans le cadre de l'approche basée sur l'usage (Bybee 2008 ; Ellis 2002) ont quant à elles mis en évidence le rôle de la fréquence des formes et des constructions dans l'input sur la production des apprenants. Elles suggèrent que la grammaire des apprenants se construise à partir de ce qu'ils trouvent dans l'input. Selon cette approche, les apprenants commencent par traiter chaque item rencontré de manière individuelle. Ce n'est qu'après une certaine expérience de la langue cible que l'apprenant fera des généralisations. Ce processus de généralisation correspond à un effet de fréquence type, que l'on peut considérer comme une forme de catégorisation. La fréquence type compte en effet le nombre d'items participants à un même pattern. Selon Paradis, Nicoladis, Crago et Genesee (2011) la fréquence type pourrait représenter la masse critique d'input qui permet la mise en place d'un schéma productif. En d'autres termes, l'apprenant doit avoir traité suffisamment de cas d'exemplaires similaire pour pouvoir en déduire un pattern ou un schéma.

Les études centrées sur les effets d'amorçage syntaxique ou structurel (structural/syntactic priming) sont ancrées dans ces deux approches en étudiant les effets de la présence ou non d'une construction sur la production ultérieure de celle-ci par les apprenants : «Syntactic priming refers to a general tendency for language users to produce a syntactic structure following previous experience with that structure. » (McDonough \& Trofimovich 2009:98). L'étude de l'effet d'amorçage structurel permet ainsi d'examiner la relation (potentielle) entre input et output dans l'interaction. Alors que le nombre d'études consacrées à l'effet d'amorçage structurel sur l'acquisition de l'anglais L2 ne cesse de croître (cf. McDonough \& Trofimovich 2009, Trofimovich \& McDonough 2011), celles consacrées au français L2 sont rares (Marsden, Altmann et St Claire 2013). En outre, et dans la ligne des études classiques de l'effet d'amorçage, la plupart des études ont été effectuées dans un cadre expérimental ou du moins fortement contrôlé. L'utilisation de corpus pour étudier l'effet d'amorçage est controversée, mais pourrait présenter un certain nombre d'avantages, notamment l'identification de facteurs interagissant sur les résultats (Gries 2005, 2011). Par ailleurs, si l'effet d'amorçage structurel est un phénomène fortement lié à l'interaction, on devrait pouvoir l'observer dans des données de corpus.

L'objectif de la présente étude est ainsi de combler un double manque : d'une part celui d'études de l'effet d'amorçage sur le français L2 et d'autre part celui d'études de l'effet d'amorçage dans des données de corpus. La présente étude se fera sur un corpus longitudinal de neuf enfants apprenants du français L2 et sur l'utilisation de l'auxiliaire être avec les verbes qui le demandent aux temps composés du passé. 


\section{Considérations théoriques}

\subsection{L'amorçage structurel}

On observe un effet d'amorçage lorsque la présentation d'un item (l'amorce) facilite le traitement ultérieur d'un item similaire (la cible). Par facilitation on entend notamment que la production de la cible sera plus rapide ou plus exacte que dans une condition sans amorce. L'amorçage syntaxique ou structurel est plus précisément un phénomène de réutilisation de la structure syntaxique d'une phrase amorce qu'un locuteur vient de percevoir ou de produire (Poletti, Le Bigot et Rigalleau 2012). En principe l'effet d'amorçage s'observe lorsque le locuteur produit la structure présentée dans l'amorce alors qu'une autre structure aurait pu être utilisée. Un exemple d'amorçage structurel est présenté en (1). Il s'agit d'un exemple typique tiré d'une étude sur l'utilisation des constructions datives en anglais, qui peuvent être exprimée soit dans l'ordre canonique he gives a present to her 'il donne un cadeau à elle' soit avec une inversion des objets he gives her a present 'il donne (à) elle un cadeau'. Comme le montre l'exemple (1) ci-dessous, l'important est la réutilisation de la structure syntaxique indépendamment de la similarité lexicale (voir Bock 1986).

(1) Exemple tiré de McDonough, 2006:182, gloses ajoutées

A: The man shows his wife the boot. 'l'homme montre à sa femme le coffre de la voiture'

B: A teacher is teaching some kids a game. 'un prof enseigne à quelques enfants un jeu'

On peut considérer que l'amorçage syntaxique est un cas d'alignement mutuel en conversation ou du moins une forme de convergence. Les raisons qui pourraient expliquer cette adaptation mutuelle sont nombreuses. Selon les premières études, conduites sur des locuteurs natifs, et d'un point de vue psychologique, l'effet d'amorçage peut s'expliquer par un processus d'activation : «An utterance takes the grammatical form that it does because the procedures controlling its syntax are more activated than the procedures responsible for an alternative form [...]. » (Bock, 1986:379). Néanmoins la nature de cette activation et sa portée sur la représentation langagière est sujet à discussion (McDonough \& Trofimovich, 2009, 104-105; Pickering \& Ferreira, 2008; Pietsch, Buch, Kopp et de Ruiter 2012). Dit simplement, il se pourrait que ce soit plus facile de réutiliser une structure syntaxique qui a été activée récemment qu'une autre structure (McDonough \& Trofimovich 2009:99). D'un point de vue pragmatique, les effets d'amorçages syntaxiques pourraient contribuer à la vitesse des échanges verbaux ainsi qu'à la compréhension mutuelle grâce à une réduction des coûts cognitifs.

\subsection{Etudes antérieures}

Que ce soit avec des locuteurs natifs (L1) ou des apprenants L2, la plupart des études sur l'effet d'amorçage structurel ont été conduites sur des apprenants de l'anglais et pour des structures telles que les constructions datives (voir ci-dessus), les phrases interrogatives et le passif. Pour ce qui est de l'acquisition d'une L2 ces études ont montré que le niveau de développement de la L2 pouvait avoir une influence sur les résultats, notamment lorsque l'une des structures cibles est plus avancée que l'autre comme c'est le cas pour les constructions datives (McDonough 2006). Il faut en effet que la structure cible soit représentée en mémoire pour qu'elle puisse être activée. En d'autres termes, l'effet d'amorçage syntaxique permet de renforcer la production d'une structure déjà présente chez l'apprenant mais pas forcément la découverte de nouvelles structures (voir McDonough et Trofimovich 2013).

Un autre facteur pouvant influencer les résultats est celui de la fréquence de la structure cible. Les résultats de l'étude de McDonough et Mackey (2008) ont montré une corrélation positive entre les effets d'amorçages syntaxiques et le développement des phrases interrogatives en anglais L2. Mais certains apprenants n'ont pas avancé vers un stade de développement plus élevé durant l'expérience. Les auteurs soulignent le rôle crucial que la fréquence type pourrait jouer « in helping learners recognize that the structure represents a general category rather than a lexically specific construction » (McDonough \& Mackey 2008:41). 
L'étude expérimentale de Marsden Altmann et St Claire (2013) est basée sur des données d'apprenants anglophones du français L2 de niveau débutant à intermédiaire. La structure ciblée était celle de la $1^{\text {ère }}$ personne du pluriel, à savoir le morphème flexionnel -ons des verbes réguliers (nous parlons). La structure a été présentée dans deux conditions différentes, une avec un focus sur la forme et l'autre avec un focus sur le sens de la phrase dans laquelle le verbe était inséré. Les résultats ont uniquement révélé un effet d'amorçage lorsque l'attention des apprenants était orientée vers la flexion. Un tel résultat suggère que le processus d'amorçage syntaxique ne se ferait pas de manière aussi implicite ou inconsciente qu'en L1 (cf. Bock et Griffin 2000), du moins en début d'acquisition.

Il existe un petit nombre d'études de l'amorçage syntaxique basé sur des données de corpus (par exemple Gérard, Keller et Palpanas 2010; Gries 2005, 2011 ; Reitter, Moore et Keller 2006). La pertinence de l'utilisation de corpus pour une telle étude est un sujet controversé. Selon Pickering et Branigan (1999) les études de corpus peuvent servir à tester des hypothèses en vue d'une étude plus contrôlée, mais les résultats obtenus ne sont pas considérés comme valables. Selon Gries $(2005,2011)$, les résultats obtenus à partir de données de corpus confirment non seulement les résultats obtenus de manière expérimentale mais permettent en outre des apports spécifiques, en particulier la détection de facteurs pouvant influencer les résultats, tels que par exemple la spécifité des verbes ou la durée de l'effet d'amorçage.

Finalement dans le domaine de l'acquisition d'une L2, l'étude de Collentine \& Collentine (2013) avec des apprenants de l'espagnol L2 sur un corpus de production écrite en ligne (chat) montre que les apprenants s'alignent au niveau syntaxique. Selon les auteurs, il s'agit d'une stratégie d'apprentissage propre à l'interaction qui pourrait être exploitée pour promouvoir l'utilisation de structures syntaxiques plus complexes.

En résumé, les études antérieures suggèrent que l'on peut trouver un effet d'amorçage syntaxique en L2, mais que le niveau des apprenants et la fréquence de la construction pourraient jouer un rôle quant à son effet positif sur le développement d'une langue seconde.

\section{Méthode}

\subsection{Structure cible}

La construction de la présente étude est basée sur les résultats d'une étude de corpus exploratoire (Thomas 2012), dans laquelle les structures syntaxiques étudiées étaient celles des verbes réguliers en -er (parler) au présent, aux temps composés du passé (auxiliaire + participe passé) et à l'infinitif (auxiliare modal + infinitif) (voir Thomas 2009 pour les structures concernées). Il s'est avéré que ces structures étaient trop fréquentes en français pour pouvoir étudier les effets d'amorçage, la production des apprenants pouvant toujours s'expliquer par un effet de fréquence (de répétition) des formes dans l'input.

Par conséquent, la structure choisie pour la présente étude est une structure bien délimitée. Il s'agit des verbes qui prennent l'auxiliaire être dans les temps composés du passé, en particulier le passé composé et le plus-que-parfait (je suis parti, elle était sortie ${ }^{l}$ ). Cette structure <auxiliare être + participe passé> est restreinte à un petit nombre de verbes de mouvement, téliques et intransitifs ainsi qu'aux verbes pronominaux. Les verbes pronominaux ne sont pas inclus dans cette étude, car leur acquisition présente des difficultés qui leur sont propres, notamment en raison du pronom réfléchi. Le tableau 1 présente les verbes concernés par cette structure ${ }^{2}$. La moitié de ces verbes sont toujours intransitifs alors que l'autre moitié présente également un usage transitif (intransitif : elle est sortie de la maison vs transitif : elle a sorti la poubelle). Les verbes instransitifs (venir...) sont donc uniquement présentés avec l'auxiliaire être dans l'input alors que les autres verbes prennent parfois l'auxiliaire avoir, qui est par ailleurs l'auxiliaire le plus fréquent dans les temps composés du passé pour les verbes français en général. La présente étude est limitée à l'utilisation de ces verbes dans leur sens intransitif uniquement. Ainsi l'exemple (2) de l'apprenante Rachel 8 (voir corpus ci-dessous) n'entre pas dans le calcul, l'amorce (et probablement la cible) correspondant à une utilisation transitive du verbe. 
*INV: d' abord pour aller dans sa chambre il a monté des escaliers .

*CHI: après il a monté parce qu' il veut manger .

\begin{tabular}{ll}
\hline Intransitifs & Intransitif et transitifs \\
\hline venir (revenir, devenir) & sortir \\
partir & (r)entrer \\
aller & monter \\
tomber & descendre \\
arriver & passer \\
rester & retourner \\
\hline
\end{tabular}

Tableau 1 : Verbes de l'étude

Le développement de la production de ces verbes par les apprenants L2 (cf. Bartning et Schlyter 2004) se fait en trois stades :

1. En début d'acquisition, les verbes aux temps composés du passé sont souvent produits sans auxiliaires : *il tombé, *il venu

2. A un niveau intermédiaire, on trouve une alternance entre les auxiliaires avoir et être : il *a venu, il est venu

3. Finalement à un niveau avancé, l'auxiliaire être est utilisé dans la plupart des cas.

$\mathrm{Au}$ deuxième stade, les apprenants ont acquis l'obligation de mettre un auxiliaire pour les temps composés du passé, mais la production de l'auxiliaire attendu est sujette à variation. Le fait que la moitié des verbes cibles de l'étude soient à la fois transitifs et intransitifs, donc que l'on trouve une variation de l'auxiliaire dans l'input, contribue probablement à cette variation chez les apprenants L2 (voir Thomas 2009 et 2014 au sujet l'influence de la variation dans l'input sur la production des apprenants).

Il est important de noter que l'utiilisation du verbe être comme auxiiliare avec les temps composés du passé n'existe pas en suédois, la L1 des apprenants (voir ci-dessous). La forme correspondant au passé composé ou au plus-que-parfait prend toujours l'auxiliaire avoir.

\subsection{Corpus}

Le corpus de cette étude comprend neuf enfants suédophones apprenants du français L2 en immersion dans une école française en Suède. Il s'agit d'une école qui suit le cursus du système scolaire français. Les données ont été collectées tous les trois mois sur une période de 2 à 3 ans dans le cadre d'un projet sur le rôle de l'âge du début d'acquisition sur le développement du français (Ågren, Granfedt, Thomas sous presse). Chaque enfant a été enregistré individuellement en principe par un adulte natif du français (parfois d'autres adultes sont intervenus pour certaines tâches). Les enregistrements font chacun environ 20 à 30 minutes. Ils comprennent tous une partie conversation sur des événements actuels, passés et futurs ainsi que des récits à partir d'images et certains comportent aussi des tests spécifiques portant par exemple sur les pronoms d'objet ou la temporalité.

Les enfants L2 ont commencé l'école maternelle ou primaire sans aucune connaissance préalable du français. A l'école, ils sont presque exclusivement exposés au français, mais aucun d'eux ne parle français à la maison. Les enfants vivent tous dans un milieu socio-économique relativement aisé.

Le tableau 2 présente les neuf enfants de l'étude. Seuls les enregistrements dans lesquels au moins un des verbes cibles (tableau 1) a été produit sont présentés (voir tableau 4). La première colonne présente le premier et le dernier enregistrement retenu et les deux dernières colonnes présentent l'âge et les mois d'exposition lors du premier et du dernier enregistrement retenu. On voit que les enfants ont un âge de 
début d'acquisition entre 3;0 et 6;7 ans, qu'ils ont entre 4;2 et 10;8 ans. Le temps d'exposition varie entre sept mois (Viola 1) à plus de trois ans d'exposition (Nancy 5).

\begin{tabular}{lcccc}
\hline $\begin{array}{l}\text { enfants L2 et } \\
\text { enregistrements }\end{array}$ & $\begin{array}{c}\text { nombre } \\
\text { d'enregistrements } \\
\text { avec verbes cibles }\end{array}$ & $\begin{array}{c}\text { âge début } \\
\text { français }\end{array}$ & $\begin{array}{c}\text { âges : } 1^{\text {er }} \text { - dernier } \\
\text { enregistrement }\end{array}$ & $\begin{array}{c}\text { mois d'exposition : } \\
1^{\text {er }} \text { - dernier } \\
\text { enregistrement }\end{array}$ \\
\hline Fia 6 & 1 & $3 ; 0$ & $4 ; 7$ & 18 \\
Naomi 3 à 10 & 3 & $3 ; 4$ & $4: 2-5 ; 6$ & $10-28$ \\
Isa 3 à 1 & 4 & $3 ; 5$ & $4 ; 3-6 ; 2$ & $10-29$ \\
Rachel 2 à 9 & 6 & $3 ; 5$ & $4 ; 2-5 ; 8$ & $9-24$ \\
Patrick 1 à 8 & 5 & $3 ; 9$ & $6 ; 3-8 ; 6$ & $17-40$ \\
Viola 1 à 11 & 10 & $6 ; 5$ & $6 ; 11-10 ; 7$ & $7-43$ \\
Hannes 2 à 10 & 7 & $6 ; 6$ & $7 ; 10-9 ; 10$ & $14-34$ \\
Valentine 2 à12 & 9 & $6 ; 7$ & $7 ; 10-10 ; 8$ & $14-44$ \\
Nancy 2 à 5 & 4 & $3 ; 4$ & $8 ; 7-9 ; 10$ & $53-64$ \\
\hline
\end{tabular}

Tableau 2 : Corpus d'enfants L2

Les transcriptions ont été faites au format CLAN (MacWhinney, 2000), dans lesquelles l'interlocuteur adulte est indiqué par *INV (interlocuteur principal) ou *ASS (assistant) et l'enfant par *CHI.

\subsection{Opérationalisation de l'effet d'amorçage structurel}

Dans les travaux à caractère expérimental ainsi que dans les études de corpus de natifs, l'étude de l'effet d'amorçage se fait de « haut en bas »: on détecte une amorce, la structure examinée, dans le locuteur 1, en suite de quoi on cherche une cible, la même structure, dans le locuteur 2. Si on trouve une cible on aura un effet d'amorçage structurel, sinon non. Dans un corpus d'apprenants L2 il est difficile d'appliquer cette méthode, le nombre d'amorces dépassant de loin le nombre de cibles. Dans cette étude, les paires amorce-cible seront mises ensemble en partant de la cible produite par l'enfant, un des verbes du tableau 1 au participe passé (indépendamment du contexte), pour ensuite chercher la présence du même verbe plus tôt chez l'interlocuteur adulte dans le même enregistrement. Cette méthode de «bas en haut » a été utilisée par Gérard, Keller et Palpanas (2010) qui ont travaillé sur un corpus d'enfants.

Une fois les paires d'amorces et de cibles obtenues, on a un contexte pour un effet d'amorçage (3) :

(3) : Contexte pour un effet d'amorçage: L'interlocuteur adulte produit une amorce avec un verbe cible (tableau 1) au participe passé précédé de l'auxiliaire être et l'enfant produit plus tard, mais dans le même enregistrement, le même verbe au participe passé, précédé ou non d'un auxiliaire, indépendamment du nombre de tours de parole ${ }^{3}$ entre les deux.

L'effet d'amorçage (4) est quant à lui défini de la manière suivante :

(4) Effet d'amorçage : Si l'enfant produit le verbe cible avec un auxiliaire être (quelle que soit sa forme ${ }^{4}$ ) dans un contexte d'amorçage, on a alors un effet d'amorçage.

Voici un exemple (5) :

(5) Viola 5 (8;7, 23 mois d'exposition)

*INV: vous êtes allés vous promener?

$<$ amorce $>$

(...8 tours)

*CHI: euh ils sont allés à \# une marché . $\quad$ <cible avec effet d'amorçage> 
Dans les cas où l'on pouvait trouver plusieurs occurrences du même verbe dans les données de l'interlocuteur adulte, la dernière avant la production de l'enfant a été considérée comme l'amorce. Inversement, une même amorce pouvait avoir plusieurs cibles.

L'établissement d'une ligne de base, qui est souvent établie à partir d'un pré-test dans les études expérimentales, ne peut pas se faire de manière indépendante dans une étude de corpus. Les préférences spontanées de l'enfant sont exprimées par les données des formes cibles produites sans amorce préalable (formes non amorcées) ${ }^{5}$.

Le fait de ne pas limiter le nombre de tours de parole risque d'augmenter le nombre d'effets d'amorçages. D'un côté on risque de compter comme effet d'amorçage un effet de répétition en raison de la faible distance entre l'amorce et la cible et de l'autre de compter une production comme amorcée alors qu'elle ne l'est peut-être plus en raison de la distance séparant les deux moments. Les études disponibles discutent cette question sans pour autant arriver à un consensus. Selon Pietsch et al. (2012) par exemple, les effets d'amorçages lexicaux seraient de courte durée alors que les effets d'amorçages syntaxiques seraient de plus longue durée. Selon Snider (2009), dans une optique de l'approche basée sur l'usage, les mécanismes sous-jacents à l'amorçage lexical et syntaxique seraient les mêmes étant donné le continuum entre lexique et grammaire. Les contextes d'amorçages de cette étude impliquant toujours le même verbe, cette hypothèse ne pourra pas être testée ici.

Finalement, il est important de noter que le choix de restreindre les contextes d'amorçage à des paires de mêmes verbes ${ }^{6}$ risque d'augmenter le nombre d'effets d'amorçage, la similiarité lexicale favorisant un tel effet (Gries 2005; Poletti, Le Bigot et Rigalleau 2012). Il nous semble néanmoins qu'à un stade où la production des auxiliaires varie pour un même verbe, ces verbes ne sont pas encore considérés comme une même catégorie de verbes mais plutôt traités individuellement. Il serait néanmoins intéressant d'élargir le champ des contextes d'amorçage dans une étude ultérieure.

\section{Questions de recherche}

L'objectif de la présente étude est de vérifier si l'on peut détecter des effets d'amorçage syntaxique dans des données de production libre d'apprenants du français L2. Les questions de recherche sont les suivantes :

1) Peut-on observer des effets d'amorçage syntaxique pour la structure des temps composés du passé avec être dans un corpus d'enfants apprenants du français L2 de niveau débutant à intermédiaire en production spontanée ?

2) Peut-on observer un développement de la production de la structure des temps composés du passé avec être dans le temps?

\section{Résultats}

Au total, les neuf enfants ont produit 228 participes passés. Comme on peut le voir dans le tableau 3, la majorité des verbes cibles ont été produits avec l'auxiliaire avoir, 124 occurrences (54\% des données), contre 86 occurrences de l'auxiliaire être (38\% des données). On voit que la préférence pour l'auxiliaire avoir dans la production non amorcée disparaît dans la production amorcée, même si le nombre d'occurrences est faible.

\begin{tabular}{llll}
\hline occurrences & $\begin{array}{l}\text { participe passé } \\
\text { seul }\end{array}$ & $\begin{array}{l}\text { auxiliaire avoir } \\
+ \text { part. passé }\end{array}$ & $\begin{array}{l}\text { auxiliaire être } \\
+ \text { part. passé }\end{array}$ \\
\hline non amorcée & 6 & 96 & 55 \\
amorcée & 12 & 28 & 31 \\
\hline
\end{tabular}

Tableau 3 : Production des participes passés par les enfants L2 
Cette augmentation de la proportion des formes des temps composés du passé avec l'auxiliaire être après amorçage est visible chez tous les enfants comme le montre le graphique 1, basé sur la production des verbes cibles avec ou sans auxiliare (seuls les enfants ayant produit au moins 10 formes au total sont présentés).

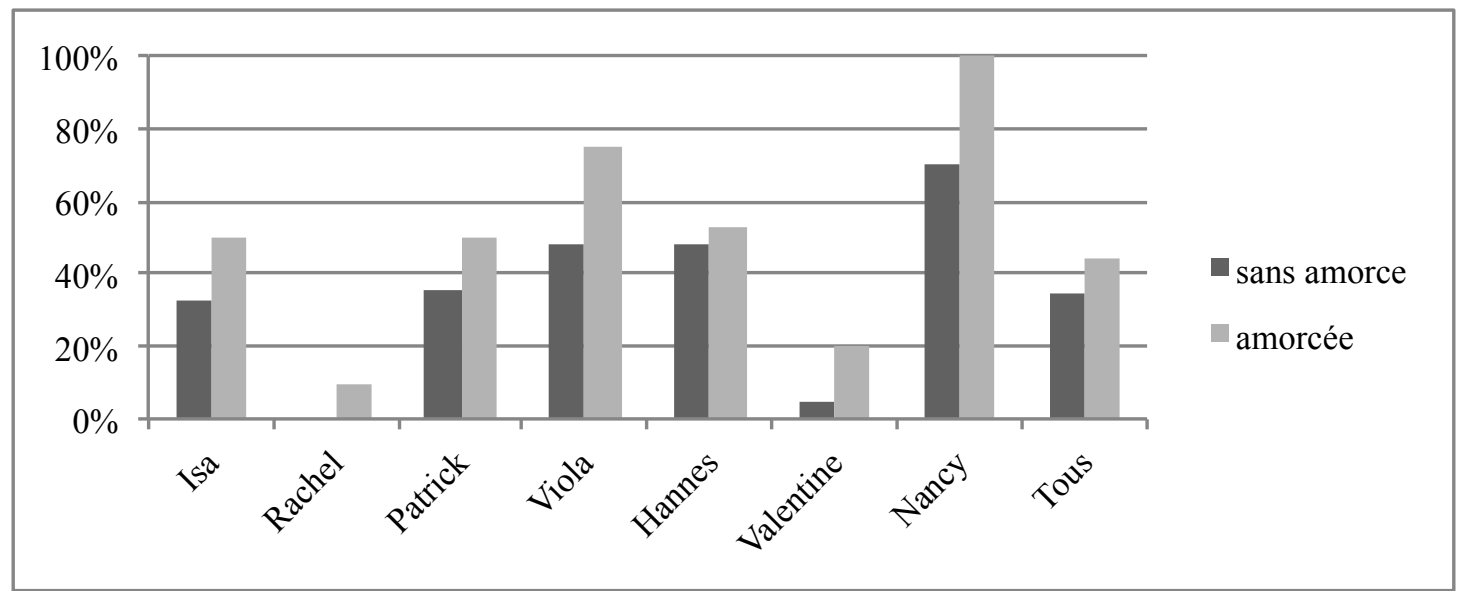

Graphique 1 : Proportion de temps composés du passé avec l'auxiliaire être

Si l'on ne tient compte que des formes produites avec un auxiliaire, la différence entre les proportions de formes produites avec les auxiliaires être et avoir dans les deux conditions est significative $\left(\mathrm{chi}^{2}=4,558\right.$, $\mathrm{p} .=.033$ ) comme illustré dans le graphique 2 . Ces résultats nous permettent de répondre positivement à la première question de recherche.

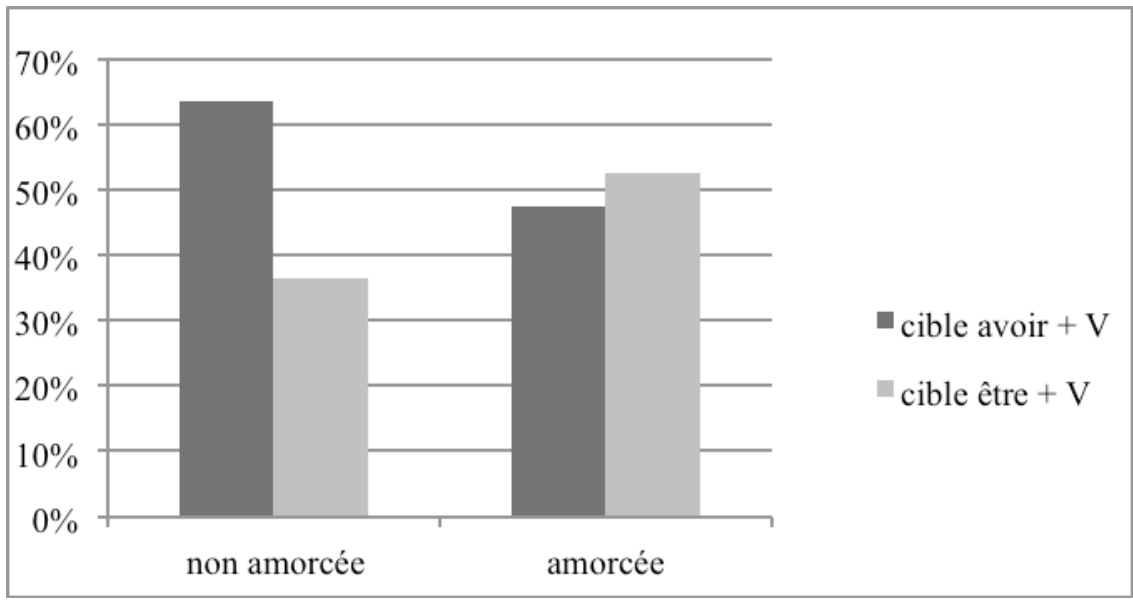

Graphique 2 : Proportion de formes composées du passé avec auxiliaire chez les enfants L2

Le graphique 3 présente une distribution de la production des apprenants répartie selon l'auxiliaire utilisé dans l'amorce de l'interlocuteur adulte. Les auxiliaires de l'amorce sont classés par ordre croissant de proportion d'auxliaires être chez les enfants L2. On voit que les formes êtes et suis sont les deux formes qui donnent lieu au plus haut taux d'effet d'amorçage. Une recherche sur la fréquence générale de ces 
deux formes dans les données des enfants L2 nous donne 37 occurrences de suis, aucune de êtes et 1040 est (ainsi que 10 es, 76 sont, 0 sommes). Il ne semble donc pas y avoir de corrélation entre les formes produites par les enfants L2 eux-mêmes et le possible effet d'amorçage de la forme.

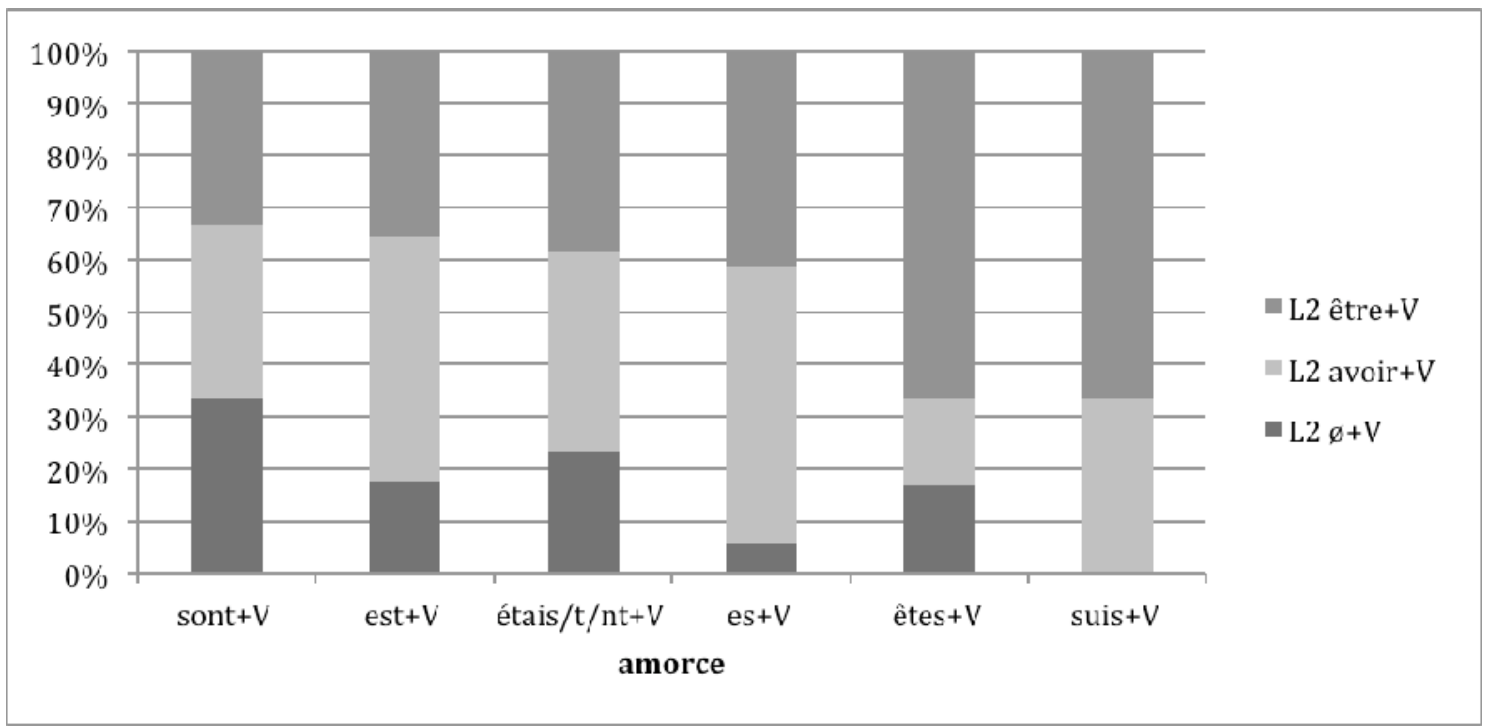

Graphique 3: Auxiliaire produit dans l'amorce et effet d'amorçage ou non

Le graphique 4 présente le nombre d'effets d'amorçages selon le nombre de tours de parole entre l'amorce et la cible. On voit que près de la moitié des effets d'amorçages (13) ont lieu durant les 10 premiers tours. La même quantité d'effets d'amorçage a ensuite lieu entre les tours 11 à 50 , après quoi on ne trouve plus que quelques effets isolés. Ce résultat confirme celui d'études antérieures, que la plupart des effets d'amorçages ont lieu rapidement, mais que l'effet peut être d'une durée assez longue (Bock et Griffin 2000).

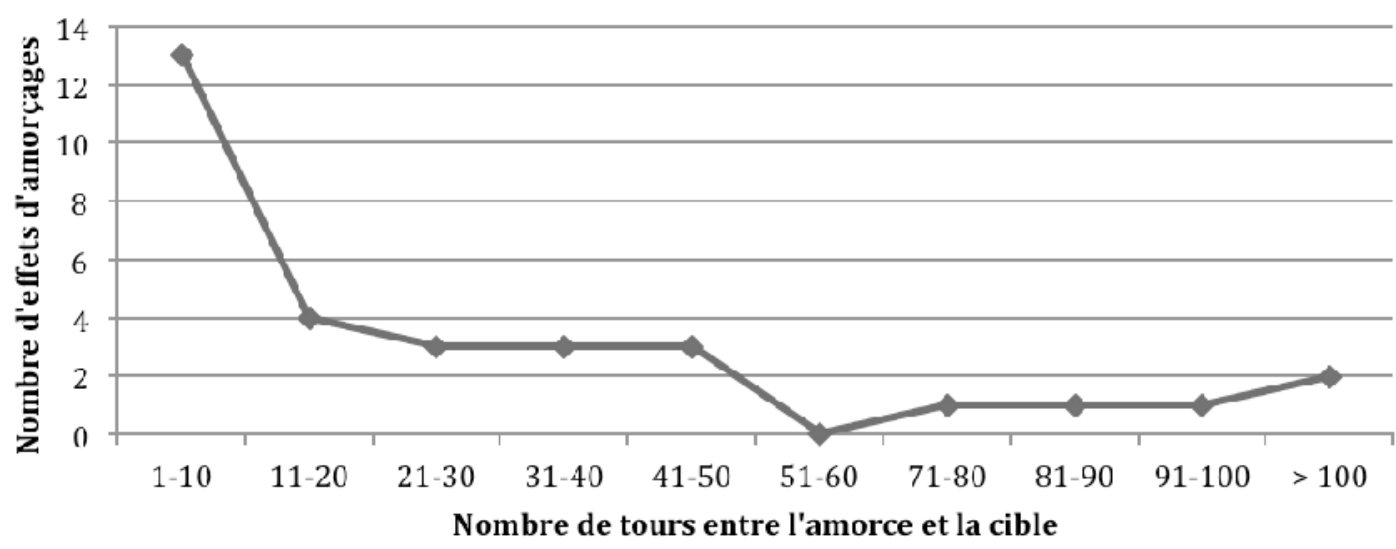

\section{Graphique 4: Distance et effet d'amorçage}


La question maintenant est de savoir si les effets d'amorçages pourraient être vus comme ayant un effet positif sur le développement de la structure examinée ici. Le tableau 4 présente les occurrences des verbes cibles (tableau 1) de manière longitudinale dans les enregistrements des enfants L2, avec d'une part le nombre d'occurrences avec l'auxiliaire être sur la somme des verbes cibles produits, puis le nombre d'effets et de contextes d'amorçage.

\begin{tabular}{lcccc}
\hline Enfants L2 & âge & $\begin{array}{c}\text { mois } \\
\text { d'exposition }\end{array}$ & $\begin{array}{c}\text { auxiliaire être } \\
\text { /occurrences verbes cibles }\end{array}$ & $\begin{array}{c}\text { effets d'amorçage } \\
\text { /nombre de contextes }\end{array}$
\end{tabular}

\begin{tabular}{|c|c|c|c|c|c|}
\hline Fia6 & $4 ; 7$ & 18 & $(1 / 1)$ & $100 \%$ & $1 / 1$ \\
\hline Naomi3 & $4 ; 2$ & 10 & $(0 / 1)$ & $0 \%$ & $0 / 1$ \\
\hline Naomi7 & $5 ; 1$ & 20 & $(0 / 2)$ & $0 \%$ & $0 / 1$ \\
\hline Naomi10 & $5 ; 6$ & 28 & $(0 / 1)$ & $0 \%$ & - \\
\hline Isa3 & $4 ; 3$ & 10 & $(2 / 3)$ & $67 \%$ & $0 / 1$ \\
\hline Isa 7 & $5 ; 2$ & 20 & $(1 / 3)$ & $33 \%$ & $1 / 1$ \\
\hline Isa9 & $5 ; 9$ & 25 & $(0 / 1)$ & $0 \%$ & - \\
\hline Isa11 & $6 ; 2$ & 29 & $(1 / 4)$ & $25 \%$ & - \\
\hline Rachel2 & $4 ; 2$ & 9 & $(0 / 2)$ & $0 \%$ & $0 / 2$ \\
\hline Rachel3 & $4 ; 3$ & 10 & $(0 / 5)$ & $0 \%$ & $0 / 4$ \\
\hline Rachel6 & $4 ; 11$ & 17 & $(0 / 2)$ & $0 \%$ & $0 / 1$ \\
\hline Rachel7 & $5 ; 2$ & 20 & $(0 / 2)$ & $0 \%$ & - \\
\hline Rachel8 & $5 ; 6$ & 22 & $(0 / 2)$ & $0 \%$ & - \\
\hline Rachel9 & $5 ; 8$ & 24 & $(1 / 3)$ & $33 \%$ & $1 / 3$ \\
\hline Patrick1 & $6 ; 3$ & 17 & $(1 / 1)$ & $100 \%$ & - \\
\hline Patrick2 & $7 ; 0$ & 24 & $(5 / 7)$ & $71 \%$ & $3 / 5$ \\
\hline Patrick3 & $7 ; 3$ & 27 & $(2 / 7)$ & $29 \%$ & $2 / 5$ \\
\hline Patrick4 & $7 ; 6$ & 30 & $(0 / 2)$ & $0 \%$ & - \\
\hline Patrick8 & $8 ; 6$ & 40 & $(1 / 4)$ & $25 \%$ & - \\
\hline Viola1 & $6 ; 11$ & 7 & $(1 / 2)$ & $50 \%$ & - \\
\hline Viola2 & $7 ; 8$ & 14 & $(3 / 5)$ & $60 \%$ & $2 / 4$ \\
\hline Viola4 & $8 ; 2$ & 20 & $(0 / 1)$ & $0 \%$ & $0 / 1$ \\
\hline Viola5 & $8 ; 7$ & 23 & $(1 / 1)$ & $100 \%$ & $1 / 1$ \\
\hline Viola6 & $8 ; 8$ & 24 & $(2 / 6)$ & $33 \%$ & - \\
\hline Viola7 & $9 ; 2$ & 30 & $(2 / 6)$ & $33 \%$ & $1 / 1$ \\
\hline Viola8 & $9 ; 6$ & 32 & $(2 / 3)$ & $67 \%$ & - \\
\hline Viola9 & $9 ; 8$ & 34 & $(2 / 3)$ & $67 \%$ & $1 / 1$ \\
\hline Viola10 & $9 ; 11$ & 37 & $(1 / 1)$ & $100 \%$ & - \\
\hline Viola11 & $10 ; 7$ & 43 & $(5 / 5)$ & $100 \%$ & $4 / 4$ \\
\hline Hannes2 & $7 ; 10$ & 14 & $(7 / 23)$ & $30 \%$ & $0 / 5$ \\
\hline Hannes4 & $8 ; 4$ & 20 & $(9 / 13)$ & $69 \%$ & $2 / 2$ \\
\hline Hannes 5 & $8 ; 9$ & 23 & $(4 / 6)$ & $67 \%$ & $1 / 1$ \\
\hline Hannes6 & $8 ; 10$ & 24 & $(3 / 6)$ & $50 \%$ & $2 / 2$ \\
\hline Hannes 7 & $9 ; 1$ & 27 & $(1 / 5)$ & $20 \%$ & - \\
\hline Hannes9 & $9 ; 8$ & 32 & $(2 / 2)$ & $100 \%$ & $2 / 2$ \\
\hline Hannes 10 & $9 ; 10$ & 34 & $(4 / 5)$ & $80 \%$ & $2 / 2$ \\
\hline
\end{tabular}




\begin{tabular}{lllccc}
\hline Enfants L2 & âge & $\begin{array}{c}\text { mois } \\
\text { d'exposition }\end{array}$ & $\begin{array}{c}\text { auxiliaire être } \\
\text { /occurrences verbes cibles } \\
\text { et \% corrects }\end{array}$ & $\begin{array}{c}\text { effets d'amorçage } \\
\text { /nombre de contextes }\end{array}$ \\
\hline Valentine2 & $7 ; 10$ & 14 & $(1 / 13)$ & $8 \%$ & $0 / 3$ \\
Valentine3 & $8 ; 1$ & 17 & $(0 / 3)$ & $0 \%$ & - \\
Valentine4 & $8 ; 9$ & 24 & $(1 / 11)$ & $9 \%$ & $1 / 6$ \\
Valentine6 & $8 ; 10$ & 25 & $(0 / 12)$ & $0 \%$ & - \\
Valentine7 & $9 ; 1$ & 28 & $(0 / 6)$ & $0 \%$ & $0 / 2$ \\
Valentine8 & $9 ; 4$ & 31 & $(1 / 5)$ & $20 \%$ & - \\
Valentine9 & $9 ; 8$ & 33 & $(0 / 2)$ & $0 \%$ & - \\
Valentine11 & $10 ; 1$ & 38 & $(0 / 1)$ & $0 \%$ & - \\
Valentine12 & $10 ; 8$ & 44 & $(2 / 6)$ & $33 \%$ & $2 / 2$ \\
\hline Nancy2 & $8 ; 7$ & 53 & $(1 / 3)$ & $33 \%$ & - \\
Nancy3 & $8 ; 11$ & 56 & $(2 / 3)$ & $67 \%$ & - \\
Nancy4 & $9 ; 5$ & 59 & $(7 / 11)$ & $64 \%$ & $1 / 1$ \\
Nancy5 & $9 ; 10$ & 64 & $(8 / 8)$ & $100 \%$ & $1 / 1$ \\
\hline
\end{tabular}

Tableau 4 : Verbes cibles avec l'auxiliaire être chez les enfants $L 2$ et nombre d'effets d'amorçage

Le tableau 4 montre que la plupart des enfants utilisent les verbes cibles de manière productive mais que la proportion d'utilisation de l'auxiliaire attendu varie selon les personnes tout comme la proportion de contextes d'amorçage.

On a en effet un groupe d'enfants qui finissent par atteindre un niveau d'utilisation de l'auxiliaire être proche de $100 \%$, Viola, Hannes et Nancy; un groupe d'enfants qui se développent tout en restant loin d'une bonne proportion de production de l'auxiliaire avec 33\% de production de l'auxiliaire, Rachel et Valentine, et finalement un groupe d'enfants qui baissent dans leur production correcte, Isa, qui passe de $67 \%$ à $25 \%$ et Patrick qui passe de $100 \%$ à $25 \%$.

Il semble que la production des temps composés du passé avec être soit particulièrement difficile pour les apprenants les plus jeunes (dans le haut du tableau) et ils semblent peu sensibles aux amorces dans l'input à l'exception d'Isa qui produit deux est tombé déjà après 10 mois d'exposition.

Nancy par contre, l'apprenante avec le plus de temps d'exposition au français, augmente à la fois le nombre d'occurrences et le taux de production de l'auxiliaire sans beaucoup d'amorces. Elle semble avoir acquis l'utilisation de l'auxiliaire être avec les verbes cibles au plus tard dans le dernier enregistrement.

Comme on l'a vu dans le graphique 1, l'apprenante Rachel ne produit pas de temps composé avec être sans amorce. Dans les premiers enregistrements cette apprenante se trouve en effet à un stade où elle ne produit pas encore d'auxiliaire et l'absence d'auxiliaire persiste longtemps dans ses données. L'exemple (6) présente la première production d'un auxiliaire avec la forme du passé composé dans les enregistrements avec Rachel. Bien qu'il n'ait pas été compté comme tel, étant donné l'absence de l'auxiliaire être, on pourrait ici avoir un effet d'amorçage pour un niveau débutant (cf. stade 1 ci-dessus).

(6) Rachel 3 (4;3, 10 mois d'exposition)

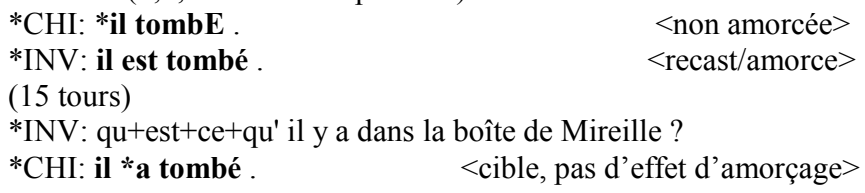

Les enfants plus âgés produisent tous les verbes cibles de manière relativement productive. La production de Patrick est caractérisée par une chute de la production de l'auxiliaire être. On peut constater que presque toutes les productions des verbes cibles est amorcée dans Patrick 2 et 3 , mais que les amorces disparaissent par la suite. Contrairement à Nancy, Patrick n'est pas sur le point de passer du stade 2 au 
stade 3 de la structure cible, mais se trouve plutôt entre les stades 1 et 2 . L'absence de contextes d'amorçage dans les derniers enregistrements pourrait expliquer le faible dévelopement de cet apprenant.

L'apprenante Viola est celle qui se développe le mieux au niveau de la production de l'auxiliaire attendu, atteignant $100 \%$ d'utilisation correcte à la fin de l'étude. Viola et Hannes sont les deux apprenants qui semblent sensibles aux amorces présentées dans l'input. Dans l'exemple (7) on voit que la production de Viola correspond typiquement à une apprenante de niveau intérmédiaire : elle hésite entre les deux auxiliaires. Elle réussit pourtant à produire l'auxiliaire attendu après une amorce. Dans cet exemple, on a l'impression que l'amorce aide l'apprenante à sortir de son hésitation.

(7) Viola 9 (9;8, 34 mois d'exposition)

*CHI: je suis allée à \# ö [/] ö Öland@s.

$<$ non amorcée $>$

(... 16 tours)

*CHI: \# *j'ai allé caresser les cheveux [\%=chevaux] qui sont à côté de ma [/] de ma \# maison. <non amorcée> (...9 tours)

*INV: okey d' accord \# mais donc est + ce + que tu es allé en France une fois dernièrement ou ? <amorce>

*CHI: oui je suis allée à France mais j' ai pas fait du cheval.

$<$ cible, effet d'amorçage $>$

Alors que Hannes finit par obtenir un assez bon pourcentage de production de l'auxiliaire être, Valentine produit assez systématiquement l'auxiliaire avoir. En fait ce n'est que lors du dernier enregistrement qu'elle commence à produire quelques occurrences de l'auxiliaire attendu. Dans les premiers enregistrementes de ces deux apprenants, les amorces de la $2^{\mathrm{e}}$ personne du singulier semblent avoir un effet négatif sur la production de l'auxiliaire. Ces deux enfants produisent en effet systématiquement j'ai... donc l'auxiliaire avoir, après l'amorce tu es... comme illustré dans l'exemple (8). L'homophonie de la forme ai du verbe avoir et es/est du verbe être pourraient en être la cause.

(8) Hannes 2 (7;10, 14 mois d'exposition)

*INV: tu es allé sur la glace?

*CHI: oui et après *j'ai allé euh un peu plus euh et sur la terre.

$<$ amorce $>$ $<$ cible, pas d'effet d'amorçage>

Hannes semble ensuite développer une stratégie qui lui permet de résoudre le problème au niveau du choix de l'auxiliaire, mais pas au niveau de la temporalité, en mettant systématiquement le verbe être à l'imparfait avec les verbes de l'étude comme illustré en (9).

(9) Hannes 4 (8;4, 20 mois d'exposition)

*INV : tu es déjà allé là-bas?

$<$ amorce $>$

(5 tours)

*CHI : j’étais allé là-bas

( $>100$ tours)

*INV: ou est+ce+que tu te rappelles [//] est+ce+que tu es déjà allé à Grönalund.

$<$ cible, effet d'amorçage >

(22 tours)

*CHI: un soir moi j'étais allé dehors parce que j'ai oublié [//] j'avais cherché quelque chose [/] quelque chose.

<cible, effet d'amorçage>

Valentine utilise une stratégie similaire, mais la forme 'défaut' qu'elle a choisi est celle du verbe avoir. L'exemple (10) présente un des rares effets d'amorçage chez Valentine. On voit qu'elle produit correctement l'auxiliaire une fois, il est venu, mais qu'elle revient ensuite à sa production idiosyncrasique.

(10) Valentine 4 (8;9, 24 mois d'exposition)

*ASS2: $<$ donc l' oiseau il est venu $>[<]$.

*ASS1: ou dès le début?

*ASS2: dès le début ou à quel moment il est venu ?

*CHI: euh, il est venu à la fin

$<$ amorce $>$

(7 tours)

$<$ cible, effet d'amorçage $>$ 
${ }^{*} \mathrm{CHI}$ : la barrière mais il avait se fait mal sur le pied [/] sur le pied et après le [//] une oiseau avait venu et (...). $<$ cible, pas d'effet d'amorçage>

Comme pour Patrick, les contextes d'amorçages dans les données de Valentine se trouvent surtout dans les premiers enregistrements, et on ne trouve pas de tel contexte dans les enregistrements 8,9 et 11 où elle produit des occurrences des verbes cibles. L'absence d'amorces dans l'input de Valentine pourrait contribuer au faible développement de cette apprenante pour cette construction grammaticale.

\section{Discussion}

L'objectif de la présente étude était de vérifier si l'on peut trouver des effets d'amorçage syntaxique dans des données de corpus. Les analyses ont été faites sur un corpus longitudinal d'enfants apprenants du français L2 pour la construction syntaxique des temps composés du passé (par exemple passé composé) avec l'auxiliaire être (voir la liste des verbes cibles dans le tableau 1).

Ces analyses ont tout d'abord permis de répondre positivement à la question de savoir si l'on pouvait observer un effet d'amorçage pour cette construction. Les données ont montré que tous les enfants produisaient plus d'auxiliaires être avec les verbes qui prennent cet auxiliaire après avoir été exposés à une amorce, comparé à une production sans amorce, et que, toutes les occurrences prises ensemble, cette différence était significative. Ces résultats confirment ainsi le fait qu'il est possible d'observer des effets d'amorçage en conversation spontanée non seulement dans des corpus de locuteurs natifs (Gries 2005, 2011) mais aussi de locuteurs L2.

Une analyse des formes de l'auxiliaire utilisées dans les amorces a montré que certaines formes d'auxiliaires donnaient lieu a plus d'effet d'amorçage que d'autres. Le fait que suis et êtes soient les auxiliaires avec le plus haut taux de succès pourrait s'expliquer en terme de saillance. Il s'agit en effet des deux seules formes du verbe être (sommes n'étant pas produit dans les amorces de ce corpus) qui peuvent considérées comme spécifiques à une forme du verbe être. Les formes es et est sont en effet homophones avec la forme ai du verbe avoir, la forme étais de l'imparfait singulier et $3^{\mathrm{e}}$ pluriel peut être difficile à séparer de la forme du participe passé été et finalement la différence entre ils ont et ils sont est difficile à faire pour les apprenants suédophones qui n'ont pas le son [z] dans leur L1. Il serait intéressant de vérifier ce résultat dans un design expérimental.

Une analyse du nombre de tours entre les amorces de l'input et les cibles produites par les enfants dans les contextes d'amorçage a montré que la plupart de ces effets avaient lieu assez rapidement mais pouvaient aller jusqu'à 50 tours au moins, confirmant ainsi la possible persistance de l'effet dans le temps (Bock et Griffin 2000; Gries 2005; Pietsch et al. 2012). Dans la littérature, cette persistance est interprétée comme l'indice d'un effet d'acquisition implicite en raison du faible niveau d'attention qu'une telle durée implique (Pickering \& Ferreira 2008).

L'examen de l'effet d'amorçage syntaxique dans un corpus longitudinal d'apprenants a également confirmé le fait que les effets d'amorçage varient selon le niveau de développement de la L2 de l'apprenant (McDonough 2006). Les effets d'amorçage pourraient aider l'apprenant à passer d'un stade débutant où les participes passés sont produits sans être précédés par un auxiliaire au stade intermédiaire où les apprenants alternent dans leur choix d'auxiliaires, comme on l'a vu avec l'apprenante Rachel dans l'exemple (6). A ce niveau, les amorces avec l'auxiliaire être, au même titre que les amorces avec l'auxiliaire avoir, permettent à l'apprenant de mettre en place la structure syntaxique des temps composés du passé. Une fois que ce niveau intermédiaire est atteint et que l'auxiliaire est le plus souvent exprimé, les amorces semblent encore une fois soutenir l'apprenant vers une production plus correcte, à savoir la production de l'auxiliaire être avec les verbes cibles. Les possibles effets bénéfiques de l'effet d'amorçage sur le développement de cette construction grammaticale semblent avant tout avoir lieu lorsque l'apprenant hésite entre les deux formes comme on l'a vu avec l'apprenante Viola dans l'exemple (7). Dans ces cas, l'effet d'amorçage pourrait avoir un effet très proche d'un effet de fréquence, un effet qui à son tour pourrait être particulièrement saillant en conversation en raison de l'alignement mutuel 
sous jacent à l'effet d'amorçage. Cette supposition pourrait être soutenue par le fait que la plupart des effets d'amorçage de la présente étude ont lieu avec le verbe aller (21 des 31 effets d'amorçage, 68\%) qui est aussi le verbe le plus souvent utilisé avec l'auxiliaire être chez les interlocuteurs du corpus (140 occurrences du verbe aller, 49 occurrences de partir, 33 occurrences de venir, les trois verbes cibles les plus fréquents dans ce corpus). Des travaux récents sur le rôle de la fréquence des formes dans l'input suggèrent qu'on trouve souvent un élément d'une catégorie donnée qui s'avère plus fréquent que les autres (cf. loi de Zipf dans Ellis et Ferreira-Junior 2009). Cet élément peut-être considéré comme le représentant de sa catégorie. Ceci pourrait être le cas du verbe aller comme représentant de la catégorie des verbes téliques intransitifs.

L'effet d'amorçage semblait avoir peu d'effet sur les apprenants les plus jeunes (à l'exception de Rachel), probablement parce qu'il leur faut plus d'expérience avec la langue cible pour pouvoir produire ces verbes au temps composés du passé. L'amorçage semblait également avoir peu d'influence sur la production de l'apprenante Nancy qui était sur le point d'avoir acquis ce phénomène au moment de commencer l'étude, sa production des verbes étant principalement non amorcée tout en étant de plus en plus correcte. Dans le cadre de l'acquisition d'une L2, l'effet d'amorçage pourrait donc surtout avoir un effet bénéfique au niveau intermédiaire, comme on l'a vu pour Viola. L'effet d'amorçage semble soutenir et renforcer le processus d'acquisition d'une structure cible. En effet, les deux enfants qui présentent le plus fort développement de la production de l'auxiliaire être avec les verbes cibles, Viola et Hannes, sont aussi les enfants qui tiennent le mieux compte des contextes d'amorçages présents dans l'input.

Inversement, on a pu voir que certains enfants de niveau intermédiaire ne se développaient pas au niveau de la structure syntaxique examinée. Chez ces enfants, Patrick et Valentine, les contextes d'amorçage n'étaient pas distribués sur la totalité des enregistrements, mais avant tout présents dans les premiers enregistrements. Le fait que l'apprenant ne tienne pas compte des contextes d'amorçages dans sa production mais persiste dans une production incorrecte ou idiosyncrasique semble ne pas promouvoir la production de nouvelles amorces. Cela pose la question de la quantité de contextes d'amorçages nécessaires ainsi que de leur timing; ils doivent arriver aux bons moments, c'est-à-dire dans des moments où l'apprenant pourrait être prêt à aller plus loin. Les mouvements d'adaptation mutuelle sur une structure syntaxique spécifique se font peut-être uniquement dans ces moments-là. Cela pourrait expliquer pourquoi Valentine n'a pas de production amorcée dans les enregistrements où elle a établi une certaine routine en utilisant la forme auxiliaire 'défaut' avais/t avec ces verbes et que les amorces ne réapparaissent que plus tard. Il serait intéressant de tester cette hypothèse de manière plus expérimentale avec des apprenants de différents niveaux.

En somme, les données indiquent une possible corrélation entre les contextes d'amorçages et le développement de la structure par les apprenants L2. Une telle corrélation pourrait souligner le caractère fortement interactif de l'effet d'amorçage, dans le sens que l'interaction permet une activité d'adaptation mutuelle.

La présente étude comporte un certain nombre de limitations, tel que le nombre d'occurrences traitées. Le fait que l'étude soit limitée à des paires de mêmes verbes a pu influencer l'obtention d'un effet général d'amorçage dans les données. Il serait intéressant d'élargir l'étude en examinant l'utilisation incorrecte de l'auxiliaire être avec d'autres verbes ou d'analyser les effets d'amorçage dans des fenêtres plus délimitées au niveau du nombre de tours mais par contre avec plus de verbes différents.

\section{Remerciements}

Je tiens à remercier les collègues du séminaire de linguistique française de l'Université de Lund pour leurs commentaires sur une version préliminaire de ce travail et en particulier Jonas Granfeldt pour sa lecture attentive du manuscrit. Je remercie également les évaluateurs anonymes pour leurs remarques pertinentes. Cette étude a notamment été rendue possible grâce au financement du Birgit Rausing Language Programme. 


\section{Références bibliographiques}

Ågren, M. Granfeldt, J. \& Thomas, A. (sous presse). Combined effects of Age of Onset and Input on the Development of Different Grammatical Structures: A Study of Simultaneous and Successive Bilingual Acquisition of French. Linguistic Approaches to Bilingualism 4(4).

Bartning, I. et Schlyter, S. (2004). Itinéraires acquisitionnels et stades de développement en français L2. Journal of French Language Studies, 14, 281-299.

Bock, K. (1986). Syntactic persistence in language production. Cognitive Psychology, 18, 355-387.

Bock, K. et Griffin, Z. (2000) The Persistence of Structural Priming: Transient Activation or Implicit Learning? Journal of experimental psychology: General, 129(2), 177-192.

Bozier, C. (2005). La sollicitation dans l'interaction exolingue en français. Thèse de doctorat, Université de Lund.

Bybee, J. (2008). Usage-based grammar and second language acquisition. In P. Robinson \& N.C. Ellis (éds), Handbook of cognitive linguistics and second language acquisition, New-York : Routledge, 216-236.

Collentine, J. et Collentine K. (2013). A corpus approach to studiyng structural convergence in task-based Spanish L2 interactions. In K. McDonough et A. Mackey (éds), Second language interaction in diverse educational contexts (pp. 167-187). Amsterdam: John Benjamins.

Ellis, N.C. (2002). Frequency effects in language processing. A review with implications for theories of implicit and explicit language acquisition. Studies in Second Language Acquisition, 24, 143-188.

Ellis, N.C. \& Ferreira-Junior, F. (2009). Construction learning as a function of frequency, frequency distribution, and function. The Modern Language Journal 93 (iii), 370-385.

Gass, S. M. (1997). Input, interaction, and the second language learner. Mahwah, NJ: Erlbaum.

Gass, S. M. (2003). Input and interaction. In C. Doughty \& M. H. Long (éds), The handbook of second language acquisition (pp. 224-255). Oxford, UK: Blackwell.

Gerard, J., Keller, F. et Palpanas, T. (2010). Corpus evidence for age effects on priming in child language. Proceedings of the 32nd Annual Meeting of the Cognitive Science Society, 218-23.

Gries, Stefan Th. (2005). Syntactic priming: A corpus-based approach. Journal of Psycholinguistic Research, 34, 365-399.

Gries, Stefan Th. (2011). Studying syntactic priming in corpora. Implications of different levels of granularity. In D. Schönefeld (éd), Converging evidence. Methodological issues for linguistic research (pp.143-164). Amsterdam: John Benjamins.

Long, M.H. (1983). Native speaker/non-native speaker conversation and the negotiation of comprehensible input. Applied Linguistics 4:2, 126-141.

Long, M. H. (1996). The role of linguistic environment in second language acquisition. In W. Ritchie \& T. Bhatia (éds.), Handbook of second language acquisition (pp. 413-468). San Diego: Academic Press.

MacWhinney, B. (2000). The CHILDES project: tools for analyzing talk. Mahwah, NJ : Lawrence Erlbaum Associates.

Marsden E., Altmann G. et St Claire M. (2013). Priming of verb inflections in L1 and L2 French: A comparison of 'redundant' versus 'non-redundant' training conditions. IRAL, 51, 271-298.

Matthey, M. (1996), Apprentissage d'une langue et interaction verbale. Sollicitation, transmission et construction de connaissances linguistiques en situation exolingue. Bern: Peter Lang.

McDonough, K. (2006). Interaction and syntactic priming. English L2 speakers' production of dative constructions. SSLA, 28, 179-207.

McDonough, K. \& Mackey, A. (2008). Syntactic priming and ESL question development. Studies in Second Language Acquisition, 30, 31-47. 
McDonough, K. \& Trofimovich, P. (2009). Using priming methods in second language research. London : Routledge.

McDonough, K. \& Trofimovich, P. (2013). Learning a novel pattern through balanced and skewed input. Bilingualism: Language and Cognition 16(3), 654-662.

Paradis, J., Nicoladis, E., Crago, M. \& Genesee F. (2011). Bilingual children's acquisition of the past tense: a usagebased approach. Journal of Child Language, 38, 554-578.

Pickering, M. et Ferreira, V. (2008). Structural priming: A critical review. Psychological Bulletin, 134(3), 427-459.

Pickering, M. et Branigan, H. (1999). The representation of verbs: Evidence from syntactic priming in language production. Journal of Memory and Language, 39, 633-651.

Pietsch, C., Buch, A., Kopp, S. et de Ruiter, J. (2012). Measuring syntactic priming in dialogue corpora. In B. Stolterfoht \& S. Featherston (éds.), Studies in Generative Grammar [SGG]: Vol. 111. Empirical Approaches to Linguistic Theory: Studies in Meaning and Structure (pp. 29-42). Berlin: Mouton de Gruyter.

Poletti, M., Le Bigot, L. et Rigalleau F. (2012). Les intérêts théoriques de l'amorçage syntaxique. L'année psychologique, 112, 247-275.

Reitter, D., Moore, J. D., \& Keller, F. (2006). Priming of syntactic rules in task-oriented dialogue and spontaneous conversation. In Proceedings of the 28th Annual Conference of the Cognitive Science Society (pp. 685-690). Vancouver, Canada: Cognitive Science Society.

Snider, N. (2009). Similarity and structural priming. In Proceedings of the 31st Annual Conference of the Cognitive Science Society (pp. 815-820). Amsterdam: Cognitive Science Society.

Thomas, A. (2009). Les apprenants parlent-ils à l'infinitif? Influence de l'input sur la production des verbes par des apprenants adultes du français. Thèse de doctorat, Université de Lund.

Thomas, A. (2012). Methodological issues in the study of priming effects in a corpus of spoken L2 French. Communication orale, Second Language Research Forum, Pittsburgh (PA), USA.

Thomas, A. (2014). Le rôle de l'aspect lexical et de la fréquence des formes dans l'input sur production des formes du passé par des enfants apprenants du français L2 en début d'acquisition, Canadian Modern Language Review $/$ La Revue canadienne des langues vivantes 70(1), 1-27.

Trofimovich, P., \& McDonough, K. (Eds.). (2011). Insights from psycholinguistics: Applying priming research to L2 learning and teaching. Amsterdam: John Benjamins.

Véronique, G.D. (2010). The Processing of Input in Second Language Acquisition/Le traitement de l'input dans l'acquisition des langues étrangères. Special issue of Language, Interaction and Acquisition, 1:2.

\footnotetext{
${ }^{1}$ Les données des interlocuteurs adultes contient une occurrence du conditionnel passé (serais allée) et une du subjonctif passé (sois partie), dans Livia 4, mais ces formes ne sont pas des amorces (il n'y a pas d'effet d'amorçage dans Livia 4).

${ }^{2}$ Les verbes naître et mourir n'ont pas été inclus; la structure il est né/mort ressemblant autant à une structure d'attribut du sujet que de passé composé.

${ }^{3}$ Un tour de parole correspond à un changement de locuteur. Dans certains enregistrements, le nombre de phrases par tour de parole chez l'interlocuteur adulte dépasse largement celui des apprenants.

${ }^{4}$ La structure cible à acquérir par les enfants est la production de l'auxiliaire être avec les verbes qui le demandent et non pas la temporalité ou l'accord sujet-verbe. Pour cette raison la forme utilisée par l'enfant L2 n'est pas considérée à part.

${ }^{5}$ Les contextes d'auto-amorçage ne sont pas considérés dans cette étude.

${ }^{6}$ Les verbes devenir et revenir ont par contre été comptés avec le verbe venir étant donné qu'il s'agit de composés de ce verbe et qu'ils sont parfois confondus par les enfants.
} 